\title{
762.
}

\section{ON SCHUBERT'S METHOD FOR THE CONTACTS OF A LINE WITH A SURFACE.}

[From the Quarterly Journal of Pure and Applied Mathematics, vol. xvII. (1881), pp. 244-258.]

I wISH to reproduce in part $\S 33$, "Coincidenz von Schnittpunkten einer Geraden mit einer Fläche" of Schubert's very interesting work Calcul der abzählende Geometrie, Leipzig, 1879, explaining in the first instance (but not altogether in the manner or from the point of view of the author) the general principles of the theory.

We have to do with conditions relating to a subject; the subject is a geometrical form or entity of any kind depending upon a certain number of constants; and the condition is onefold, twofold, \&c., according as it imposes a onefold, twofold, \&c., relation upon these constants. The number of constants is the Postulandum of the subject, and the manifoldness of the condition is called also its Postulation. A condition is incomplete when its postulation is less than postulandum of subject, complete when its postulation is equal to postulandum of subject; two or more incomplete conditions, making up a complete condition, are supplementary to each other. The case where the postulation exceeds the postulandum, or say that of a more than complete condition, is not in general considered; it may however sometimes present itself. For instance, the subject may be a line with $n$ points upon it; the number of constants is here $=n+4$. A condition that the line shall meet a given line, or that a certain one of the $n$ points shall lie on a given plane, is a onefold condition; the condition that such point shall lie upon a given line is a twofold condition; and so in other cases.

Conditions are denoted by letters, and simultaneous conditions by a product; for instance, the subject is a line carrying the $n$ points $1,2, \ldots, n ; g$ is the condition that the line meets a given line; $p_{1}$ the condition that the point 1 lies on a given plane; then $g p_{1}$ is the twofold condition that the line meets a given line and that C. XI. 
the point 1 lies on a given plane; $p_{1}{ }^{2}$ is the twofold condition that the point 1 lies on each of two given planes (in fact, on their line of intersection). The letters $p, g, e$ are used as the initials of Punkt, Gerade, Ebene.

The letter or combination of letters denoting an incomplete condition, or, say, the incomplete condition itself, has no numerical value; but for a complete condition there exists a definite number of subjects satisfying the condition, and the condition is regarded as having this number as its value. A more than complete condition has the value 0 .

Conditions of the same postulation may be connected by the sign + ; for instance, subject a line,

$g_{e}$ the condition that it lies in a given plane,

$g_{p}$ the condition that it passes through a given point,

then $g_{e}+g_{p}$ is the condition that the line shall either lie in the given plane or else pass through the given point.

I abstain from attempting any definition in regard to the sign -.

Conditions of the same postulation may be connected by an equation or equations; for instance,

subject a point,

$p$ the condition that the point shall lie in a given plane,

$p_{g}$ the condition that the point shall lie in a given line,

then $p^{2}=p_{g}$.

This equation has (so far) no numerical signification; it has the logical signification that the condition that a point shall lie on each of two given planes is equivalent to the condition that the point shall lie on a given line.

Second example. Subject a line,

$g$ the condition that the line meets a given line,

$g_{e}$ the condition that it lies in a given plane,

$g_{p}$ the condition that it passes through a given point,

then $g^{2}=g_{e}+g_{p}$.

This equation has (so far) no numerical signification, and I regard it as having no logical signification. Schubert, however, gives it a logical signification by means of his "Princip der speciellen Lage" (Principle of Special Situation), viz. the condition of the line meeting each of two given lines is, in the particular case where the two given lines meet, equivalent to the condition, that the line shall either lie in the plane of the two given lines or else pass through their point of intersection. 
Third example. Subject a line bearing upon it the points 1 and 2 ,

$\epsilon$ the condition of the coincidence of the two points,

$$
\begin{aligned}
& p \quad \text { that the point } 1 \text { shall lie on a given plane, } \\
& q \quad \text { " } \quad \text { that the line shall meet a given line, } \\
& g \quad \text { " } \\
& \text { then } \epsilon=p+q-g .
\end{aligned}
$$

This equation has (so far) no numerical signification, and it does not appear to have any logical signification. In fact, in the actual form of the equation we have a sign - which has not had given to it any logical interpretation; and if we write the equation in the form $\epsilon+g=p+q$, there seems to be no logical signification in the assertion, the condition that either the points shall coincide, or else the line meet a given line, is equivalent to the condition that either the first point, or else the second point, shall lie in a given plane.

Any equation connecting complete conditions is a numerical equation; and to render a condition complete, we have only to join to it a supplementary condition $X$ of the proper postulation. Thus, in the last example the postulandum is $=6 ; \epsilon, p$, $q, g$ are onefold conditions, and joining to each of them one and the same fivefold condition $X$, we have $X \epsilon=X p+X q-X g$. And, taking $X$ to be an arbitrary fivefold condition, the original equation $\epsilon=p+q-g$ has in fact the meaning

$$
X \epsilon=X p+X q-X g \text {. }
$$

For instance, the fivefold condition $X$ may be that the line shall belong to a given regulus (scroll or developable surface), and that the points 1,2 upon the line shall be the intersections of the line with given surfaces $S_{1}, S_{2}$ respectively. The subject is the line of the given regulus with its two points; and the meaning of the equation is that the number of subjects with two coincident points is equal to the number of subjects with the point 1 on a given plane, plus the number of subjects with the point 2 on a given plane, minus the number of subjects for which the line meets a given line. Although for the moment concerned only with the meaning of the theorem, not with its truth, I stop to show $\grave{d}$ posteriori that the theorem is in fact true: take $k$ for the order of the regulus; $m_{1}, m_{2}$ for the orders of the surfaces $S_{1}, S_{2}$ respectively; then it is to be shown that $X \epsilon, X p, X q, X g$ are each $=k m_{1} m_{2}$ (values which satisfy the equation). First $X \epsilon$ : the points 1 and 2 here coincide at a point of the curve of the order $m_{1} m_{2}$, which is the intersection of $S_{1}$ and $S_{2}$; the regulus meets this curve in $k m_{1} m_{2}$ points, and through each of these we have a line of the regulus having upon it the two coincident points; that is, $X \epsilon=k m_{1} m_{2}$. Next $X_{p}$ : the point 1 is here on the plane curve of the order $m_{1}$, which is the intersection of $S_{1}$ with the corresponding given plane; the regulus meets this plane curve in $\mathrm{km}_{1}$ points; through each of these we have a line of the regulus intersecting $S_{2}$ in $m_{2}$ points, any one of which may be taken for the point 2 ; that is, the number of subjects is $X p,=k m_{1} \cdot m_{2}$. Then $X q$ : in precisely the same manner we have $X q=k m_{2}, m_{1}$. Lastly $X g$ : the given line meets the regulus in $k$ points, and 
through each of these there is a line of the regulus meeting $S_{1}$ in $m_{1}$ points, any one of which may be taken for the point 1 , and meeting $S_{2}$ in $m_{2}$ points, any one of which may be taken for the point 2 ; the number of the subjects $X g$ is thus $X g,=k \cdot m_{1} \cdot m_{2}$.

The general theorem $X_{\epsilon}=X p+X q-X g$ is proved by means of Chasles' theorem of united points as follows: the subject is a line, or say, for convenience, an axis $\xi$, bearing upon it the two points 1 and 2 ; we consider in conjunction therefore a given line $\lambda$, and through this draw the planes $P_{1}, P_{2}$ passing through the points 1 and 2 respectively.

Suppose that when 2 lies in a given plane there are $\alpha^{\prime}$ positions of the axis, and on each of these $\beta^{\prime}$ positions of the point 1 ; and, similarly, that when 1 lies on a given plane there are $\alpha$ positions of the axis, and on each of these $\beta$ positions of the point 2 ; then, 1 lying in a given plane, the number of subjects is $\alpha \beta$, or we have $X p=\alpha \beta$; and, similarly, $X q=\alpha^{\prime} \beta^{\prime}$. Take now for the point $P_{1}$ an arbitrary plane through $\lambda$; then, 1 lying on this plane, the number of the points 2 is $=\alpha \beta$, or, since each of these determines with $\lambda$ a position of the plane $P_{2}$, the number of these planes is $=\alpha \beta$, that is, it is $=X p$; and, similarly, taking $P_{2}$ an arbitrary plane through $\lambda$, the number of the planes $P_{1}$ is $\alpha^{\prime} \beta^{\prime}$, that is, it is $=X q$; viz. the two planes $P_{1}, P_{2}$ through the line $\lambda$ have an $(X p, X q)$ correspondence; hence, by Chasles' theorem, the number of united planes is $=X p+X q$.

But we have a united plane, $1^{\circ}$, if the points 1 and 2 coincide, that is, if the condition $X \epsilon$ be satisfied, and the number of these united planes is $X_{\epsilon} ; 2^{\circ}$, if the axis $\xi$ meet the arbitrary line $\lambda$, that is, if the condition $X g$ be satisfied, and the number of these united planes is $=X g$; hence the whole number is $=X \epsilon+X g$; or we have $X p+X q=X \epsilon+X g$, that is, $X \epsilon=X p+X q-X g$, which is the theorem in question.

The conclusion is that the equation $\epsilon=p+q-g$, which in this, its original form, has neither a numerical nor a logical signification, is to be understood as meaning the numerical equation $X \epsilon=X p+X q-X g$, the truth of which numerical equation has just been proved. Or we may, without explicit introduction of the condition $X$, understand the equation $\epsilon=p+q-g$ as a numerical equation as follows, viz. taking for the subject a line with two points, which line and points are regarded as satisfying a given fivefold condition, then

$\epsilon$ is the (additional onefold) condition that the two points shall coincide,

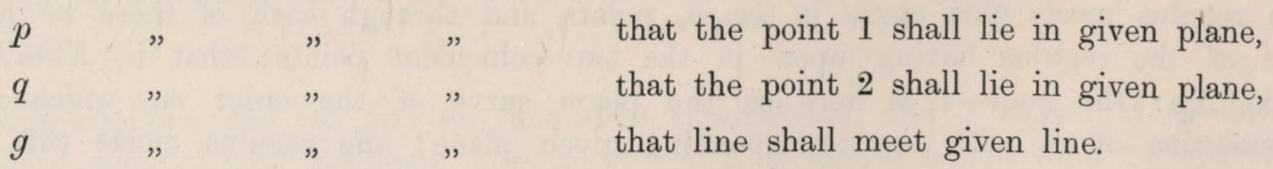

The conditions $\epsilon, p, q, g$ are thus in effect complete conditions, having values which may be connected by an equation; there, in fact, exists between them the relation

$$
\epsilon=p+q-g \text {. }
$$


The like remarks would apply to the before-mentioned equation (subject a point) $p^{2}=p_{g}$ : either adding to it a onefold condition $X$, and so taking it in the form $X p^{2}=X p_{g}$, or understanding it in its original form $p^{2}=p_{g}$ as belonging to a point which satisfies already a onefold condition, the equation is true as a numerical equation; and this in fact follows at once from its truth as a logical equation. But observe the difference: the equation in question $p^{2}=p_{g}$ has, the equation $\epsilon=p+q-g$ has not, a logical signification.

I regard as the fundamental notion of the theory the existence of equations between conditions such as the foregoing equation $\epsilon=p+q-g$; equations which in their original form have not (of necessity) any logical signification, and have not any numerical signification; but which, when we adjoin to them a supplementary condition $X$ of the proper postulation, become numerical equations, which are true, independently of the form of the supplementary condition $X$ and whatever this condition may be. And this being so, it seems to follow at once that such equations may be treated and worked with as ordinary algebraical equations. For instance, let $M$ be any condition of less postulation than $X$ : then if from the equation $\epsilon=p+q-g$, assumed to be true, we deduce $M \epsilon=M p+M q-M g$, this (like the original equation $\epsilon=p+q-g$ ) is in its actual form an equation without logical or numerical signification; but if we adjoin to it a supplementary condition $K$, such that postulation of $K+$ do. of $M=$ do. of $X$ (or, what is the same thing, that the condition $K M$ shall be supplementary to the several conditions contained in the original equation $\epsilon=p+q-g$ ), then the equation in question, $M \epsilon=M p+M q-M g$, is to be interpreted as meaning

that is,

$$
K M \epsilon=K M p+K M q-K M g,
$$

$$
X \epsilon=X p+X q-X g,
$$

which is numerically true. We thus see that the original equation $\epsilon=p+q-g$ implies the new equation

$$
M \epsilon=M p+M q-M g,
$$

which is its algebraical consequence. And if we regard, for instance, $A+B$ as the condition that either the condition $A$ shall be satisfied or else the condition $B$ shall be satisfied, then $A+B$ is a condition, and as such we have

$$
(A+B) \epsilon=(A+B) p+(A+B) q-(A+B) \epsilon .
$$

It is going a step further to say that if we have, for instance, an equation $M=A+B-C$ between conditions $M, A, B, C$, then that, instead of

we may write

$$
M \epsilon=M p+M q-M \epsilon,
$$

$$
(A+B-C) \epsilon=(A+B-C) p+(A+B-C) q-(A+B-C) \epsilon
$$

this, is, in fact, treating $A+B-C$ as being to all intents and purposes a condition such as $M$, or an alternative condition $A+B$. It is, in fact, assumed that the step is permissible; and we thus make such deductions as

$$
(\epsilon+p+q-g)(\epsilon-p-q+g)=0 ;
$$


that is,

or

$$
\epsilon^{2}-(p+q-g)^{2}=0
$$

$$
\epsilon^{2}=(p+q-g)^{2},=p^{2}+2 p q+q^{2}-2 p g-2 q g+g^{2} ;
$$

viz. this is an equation such as the original equation $\epsilon=p+q-g$, acquiring a numerical signification when we adjoin to it a supplementary condition $X$ of the proper postulation.

The section above referred to deals with the question to determine the number of lines which satisfy the several relations of contact in regard to a given surface $F$ of the order $n$, without point-singularities, that is, the surface represented by the general equation $\left(* \gamma(x, y, z, w)^{n}=0\right.$.

The chief results are contained in the following table, the notation of which will be explained :

$$
\begin{aligned}
& \text { 1. } \epsilon_{2} g_{s}=n(n-1) \text {, } \\
& \text { 2. } \epsilon_{2} b_{2} g_{e}=n \text {, } \\
& \text { 3. } \epsilon_{3} g_{e}=3 n(n-2) \text {, } \\
& \text { 4. } \epsilon_{3} g_{p}=n(n-1)(n-2) \text {, } \\
& \text { 5. } \epsilon_{3} b_{3}{ }^{2}=2 n \text {, } \\
& \text { 6. } \epsilon_{22} g_{e}=\frac{1}{2} n(n-2)(n-3)(n+3) \text {, } \\
& \text { 7. } \epsilon_{22} g_{p}=\frac{1}{2} n(n-1)(n-2)(n-3) \text {, } \\
& \text { 8. } \epsilon_{22} b_{2}{ }^{2}=n(n-3)(n+2) \text {, } \\
& \text { 9. } \epsilon_{22} b_{2} c_{2}=n\left(n^{3}-2 n^{2}+2 n-6\right) \text {, } \\
& \text { 10. } \epsilon_{4} g=2 n(n-3)(3 n-2) \text {, } \\
& \text { 11. } \epsilon_{4} b_{4}=n(11 n-24) \text {, } \\
& \text { 12. } \epsilon_{32} g=n(n-3)(n-4)\left(n^{2}+6 n-4\right) \text {, } \\
& \text { 13. } \epsilon_{32} b_{3}=n(n-4)\left(3 n^{2}+5 n-24\right) \text {, } \\
& \text { 14. } \epsilon_{32} b_{2}=n(n-2)(n-4)\left(n^{2}+2 n+12\right) \text {, } \\
& \text { 15. } \epsilon_{222} g=\frac{1}{3} n(n-3)(n-4)(n-5)\left(n^{2}+3 n-2\right) \text {, } \\
& \text { 16. } \epsilon_{222} b_{2}=\frac{1}{2} n(n-2)(n-4)(n-5)\left(n^{2}+5 n+12\right) \text {, } \\
& \text { 17. } \epsilon_{5}=5 n(n-4)(7 n-12) \text {, } \\
& \text { 18. } \epsilon_{42}=2 n(n-4)(n-5)(n+6)(3 n-5) \text {, } \\
& \text { 19. } \epsilon_{33}=\frac{1}{2} n(n-4)(n-5)\left(n^{3}+3 n^{2}+29 n-60\right) \text {, } \\
& \text { 20. } \epsilon_{322}=\frac{1}{2} n(n-4)(n-5)(n-6)\left(n^{3}+9 n^{2}+20 n-60\right) \text {, } \\
& \text { 21. } \epsilon_{2222}=\frac{1}{12} n(n-4)(n-5)(n-6)(n-7)\left(n^{3}+6 n^{2}+7 n-30\right) \text {, } \\
& \text { 22. } \epsilon_{222} b_{1}=\frac{1}{3} n(n-4)(n-5)(n-6)\left(n^{3}+3 n^{2}-2 n-12\right) \text {, } \\
& \text { 23. } \epsilon_{3} b_{1}{ }^{2}=(n-3)\left(n^{2}+2\right) \text {, } \\
& \text { 24. } \epsilon_{2} b_{1} c_{1} d_{1}=n^{2}(n-4)\left(2 n^{2}-3 n-3\right) \text {. }
\end{aligned}
$$


In the foregoing formulæ the suffixes of the $\epsilon$ refer to the contacts, viz. $\epsilon_{2}$ denotes a 2-pointic intersection, $\epsilon_{32}$ a 3-pointic and a 2-pointic intersection. The letters $b, c, d$ refer to the points of contact or intersection, thus $\epsilon_{32} b_{3}, b_{3}$ is the point of 3-pointic intersection; $\epsilon_{222} b_{1}, b_{1}$ is one of the points of simple intersection; $b_{1}$ is also the condition that the point in question lies on a given plane; $g, g_{s}, g_{e}, g_{p}$ have their ordinary signification explained a little further on. Thus (15) $\epsilon_{222} g$ denotes the number of triple tangents which can be drawn to meet a given line; or, what is the same thing, it is the order of the regulus formed by the triple tangents.

The following are elementary formulæ used in the investigation of the foregoing results.

Subject a line having upon it a point,

$p$ the condition that point is in a given plane

Postul.

\begin{tabular}{|c|c|c|c|}
\hline$p_{g}$ & , & & " line \\
\hline$g$ & $"$ & line & meets a given line \\
\hline$g_{e}$ & ” & , & is in a given plane \\
\hline$g_{p}$ & $"$ & $"$ & passes through a given point \\
\hline$g_{s}$ & $"$ & $"$ & $\begin{array}{l}\text { lies in a given plane and passes through a } \\
\text { given point of that plane }\end{array}$ \\
\hline G & ", & ” & coincides with a given line \\
\hline
\end{tabular}

We have (p. 22 et seq.)

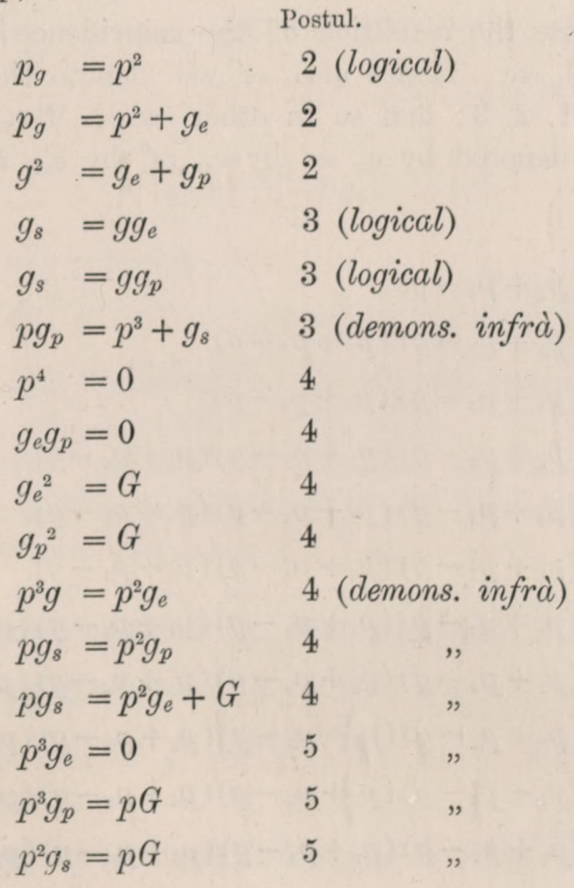


$p g_{p}=p^{3}+g_{s} ;$ we have $0=g_{e}+p^{2}-p g, 0=g_{e}+g_{p}-g^{2}$, and thence

$$
\begin{aligned}
0= & (p+g)\left\{g_{e}+p(p-g)\right\}-p\left\{g_{e}+g_{p}-g^{2}\right\} \\
= & p g_{e}+g g_{e}+p^{3}-p g^{2} \\
& -p g_{e}-p g_{p}+p g^{2} \\
= & g g_{e}-p g_{p}+p^{3} \\
= & g_{s}-p g_{p}+p^{3} .
\end{aligned}
$$

$p^{3} g=p^{2} g_{e}:$ from $p g=p^{2}+g_{e}$, we have $p^{2} g=p^{4}+p^{2} g_{e}=p^{2} g_{e}$, since $p^{4}=0$,

$$
\begin{array}{llll}
p g_{s}=p^{2} g_{e}+G, & g_{s}=g g_{e} & \# & p g_{e}=p g g_{e}=\left(p^{2}+g_{e}\right) g_{e}=p^{2} g_{e}+G, \\
p g_{s}=p^{2} g_{p} \quad, & g_{s}=g g_{p} & \# & p g_{s}=p g g_{p}=\left(p^{2}+g_{e}\right) g_{p}=p^{2} g_{p}, \text { since } g_{e} g_{p}=0 ;
\end{array}
$$

and in a similar manner we prove the last three equations.

For the demonstration of the formulæ of the table we take the subject to be a line bearing upon it the points $1,2, \ldots, n$, which are its intersections with a given surface of the order $n$. The symbols $p_{1}, p_{2}, \ldots$ refer to these points respectively; thus, $p_{1}$ is the condition that the point 1 may lie on a given plane; and then, writing

$$
\begin{aligned}
& \epsilon=p_{1}+p_{2}-g, \\
& \epsilon^{\prime}=p_{1}+p_{3}-g, \\
& \epsilon^{\prime \prime}=p_{3}+p_{4}-g,
\end{aligned}
$$

it appears that $\epsilon$ will denote the condition of the comcidence of the points 1 and 2 ; $\epsilon^{\prime}$ that of the points 1 and 3 , \&c. Hence also, $\epsilon \epsilon^{\prime}$ will denote the twofold condition of the coincidence of the points $1,2,3$; and so in other cases. But, according to the notation above explained, $\epsilon$ is also denoted by $\epsilon_{2}, \epsilon \epsilon^{\prime}$ by $\epsilon_{3}, \epsilon \epsilon^{\prime \prime}$ by $\epsilon_{22}$, \&c.

We thus have

$$
\begin{aligned}
\epsilon_{2} & =p_{1}+p_{2}-g, \\
\epsilon_{3} & =\left(p_{1}+p_{2}-g\right)\left(p_{1}+p_{3}-g\right), \\
2 \epsilon_{22} & =\left(p_{1}+p_{2}-g\right)\left(p_{3}+p_{4}-g\right), \\
\epsilon_{4} & =\left(p_{1}+p_{2}-g\right)\left(p_{1}+p_{3}-g\right)\left(p_{1}+p_{4}-g\right), \\
\epsilon_{32} & =\left(p_{1}+p_{2}-g\right)\left(p_{1}+p_{3}-g\right)\left(p_{4}+p_{5}-g\right), \\
6 \epsilon_{222} & =\left(p_{1}+p_{2}-g\right)\left(p_{3}+p_{4}-g\right)\left(p_{5}+p_{6}-g\right), \\
\epsilon_{5} & =\left(p_{1}+p_{2}-g\right)\left(p_{1}+p_{3}-g\right)\left(p_{1}+p_{4}-g\right)\left(p_{1}+p_{5}-g\right), \\
\epsilon_{42} & =\left(p_{1}+p_{2}-g\right)\left(p_{1}+p_{3}-g\right)\left(p_{1}+p_{4}-g\right)\left(p_{5}+p_{6}-g\right), \\
2 \epsilon_{33} & =\left(p_{1}+p_{2}-g\right)\left(p_{1}+p_{3}-g\right)\left(p_{4}+p_{5}-g\right)\left(p_{4}+p_{6}-g\right), \\
2 \epsilon_{322} & =\left(p_{1}+p_{2}-g\right)\left(p_{1}+p_{3}-g\right)\left(p_{4}+p_{5}-g\right)\left(p_{6}+p_{7}-g\right), \\
24 \epsilon_{2222} & =\left(p_{1}+p_{2}-g\right)\left(p_{3}+p_{4}-g\right)\left(p_{5}+p_{6}-g\right)\left(p_{7}+p_{8}-g\right) .
\end{aligned}
$$


We can now, by a mere analytical process of development and reduction, express each of the foregoing values as a linear function of

$$
p_{1}^{2} p_{2}^{2}, p_{1}^{2} p_{2} p_{3}, p_{1} p_{2} p_{3} p_{4} \text {, and } G \text {. }
$$

(Schubert says, as a linear function of these four symbols and $p_{1} p_{2} g_{e}$; but in fact $p_{1} p_{2} g_{e}$ is $=p_{1}^{2} p_{2}^{2}$.)

Observe, first, that we may, p. 287, in all the general equations instead of $p$ write $p_{1}, p_{2}$, \&c. ; and, further, that any symbol containing for instance $p_{1}{ }^{3}$ is $=0$. For the symbols now belong to the intersections of the line with a given surface; $p_{1}{ }^{3}$ is the condition that a certain one of these intersections shall lie in three given planes, that is, that it shall coincide with a given arbitrary point; this cannot be the case, for the arbitrary point is not on the surface $F$; and therefore $p_{1}{ }^{3}=0$.

We thus have $p_{1} g=p_{1}{ }^{2}+g_{e}$, thence $p_{1}{ }^{2} g=p_{1}{ }^{3}+p_{1} g_{e}$, that is, $p_{1}^{2} g=p_{1} g_{e}$; and thence further $p_{1}^{3} g=p_{1}^{2} g_{e}$, that is, $p_{1}^{2} g_{e}=0$.

Again, from $p_{2} g=p_{2}{ }^{2}+g_{e}, p_{1} g=p_{1}{ }^{2}+g_{e}$, we have

$$
p_{1}^{2}\left(p_{2}^{2}+g_{e}\right)=p_{1} p_{2}\left(p_{1}^{2}+g_{e}\right)
$$

which, in virtue of $p_{1}^{2} g_{e}=0$ and $p_{1}^{3} p_{2}=0$, becomes

$$
p_{1}^{2} p_{2}^{2}=p_{1} p_{2} g_{e} .
$$

As a simple instance of the reductions, take

Here

$$
\epsilon_{2} g_{8},=\left(p_{1}+p_{2}-g\right) g_{8} .
$$

and

$$
p_{1} g_{8},=p_{2} g_{8},=p_{1}^{2} g_{e}+G,=G \text {, since } p_{1}^{2} g_{e}=0 ;
$$

$$
g g_{s}=g^{2} g_{e}=\left(g_{e}+g_{p}\right) g_{e}=g_{e}^{2}+g_{e} g_{p}=G \text {, since } g_{e}^{2}=0, g_{e} g_{p}=G ;
$$

whence the value is

$$
\epsilon_{2} g_{8}=G+G-G,=G .
$$

As a more complicated example, take

$$
\epsilon_{5},=\left(p_{1}+p_{2}-g\right)\left(p_{1}+p_{3}-g\right)\left(p_{1}+p_{4}-g\right)\left(p_{1}+p_{5}-g\right) .
$$

Observe that, after the multiplication is effected we may, in any way we please, interchange the suffixes, $p_{1}^{2} p_{3} p_{4}=p_{1}^{2} p_{2} p_{3}, p_{3}^{2} p_{4}^{2}=p_{1}^{2} p_{2}{ }^{2}$, \&c.; the suffixes serve only to distinguish from each other symbols in the same product (thus $p_{1}{ }^{4}$ is different from $\left.p_{1} p_{2} p_{3} p_{4}\right)$, but there is nothing to distinguish one point of intersection from another. Thus the foregoing expression containing the terms $\left(p_{2}+p_{3}+p_{4}+p_{5}\right)\left(p_{1}-g\right)^{3}$, these may be combined into the single term $4 p_{2}\left(p_{1}-g\right)^{3}$; expanding in powers of $p_{1}-g$ and reducing in this manner, the value of $\epsilon_{5}$ is, in fact, found to be

$$
=\left(p_{1}-g\right)^{4}+4 p_{2}\left(p_{1}-g\right)^{3}+6 p_{2} p_{3}\left(p_{1}-g\right)^{2}+4 p_{2} p_{3} p_{4}\left(p_{1}-g\right)+p_{1} p_{2} p_{3} p_{4} \text {. }
$$

Developing this in powers of $g$, omitting the terms containing $p_{2}{ }^{3}$ which vanish, and further reducing, the value is

$$
6 p_{1}^{2} p_{2} p_{3}+5 p_{1} p_{2} p_{3} p_{4}+g\left(-12 p_{1}^{2} p_{2}-16 p_{1} p_{2} p_{3}\right)+g^{2}\left(6 p_{1}^{2}+18 p_{1} p_{2}\right)-8 p_{1} g^{3}+g^{4}
$$

C. XI. 
We have

$$
g^{4}=2 G, p_{1} g^{3}=p_{1} g_{s}=p_{1}^{2} g_{e}+G,=G .
$$

Next for the terms in $g^{2}$, from $p_{1} g=p_{1}{ }^{2}+g_{e}$ we have

and thence

$$
\begin{aligned}
& p_{1}^{2} g=\quad p_{1} g_{e}, \\
& p_{1} p_{2} g=p_{1}^{2} p_{2}+p_{1} g_{e}, \\
& p_{1}^{2} g^{2}=\quad p_{1} g_{s} ; \\
& p_{1} p_{2} g^{2}=p_{1}^{2} p_{2} g+p_{1} g_{s} \text {, }
\end{aligned}
$$

or, since $p_{1} g_{s}=G$ as before, the whole term is $=18 p_{1}^{2} p_{2} g+24 G$. The terms in $g$ thus become $=g\left(6 p_{1}^{2} p_{2}-16 p_{1} p_{2} p_{3}\right)$, and from the same equation $p_{1} g=p_{1}^{2}+g_{e}$ we find

$$
p_{1}^{2} p_{2} g=p_{1}^{2} p_{2}^{2} \text { and } p_{1} p_{2} p_{3} g=p_{1}^{2} p_{2} p_{3}+p_{1}^{2} p_{2}^{2} \text {. }
$$

The value is thus finally found to be

$$
=-10 p_{1}^{2} p_{2}^{2}-10 p_{1}^{2} p_{2} p_{3}+5 p_{1} p_{2} p_{3} p_{4}+10 G .
$$

The whole series of like results is

$$
p_{1}^{2} p_{2}^{2} \quad p_{1}^{2} p_{2} p_{3} \quad p_{1} p_{2} p_{3} p_{4} \quad G
$$

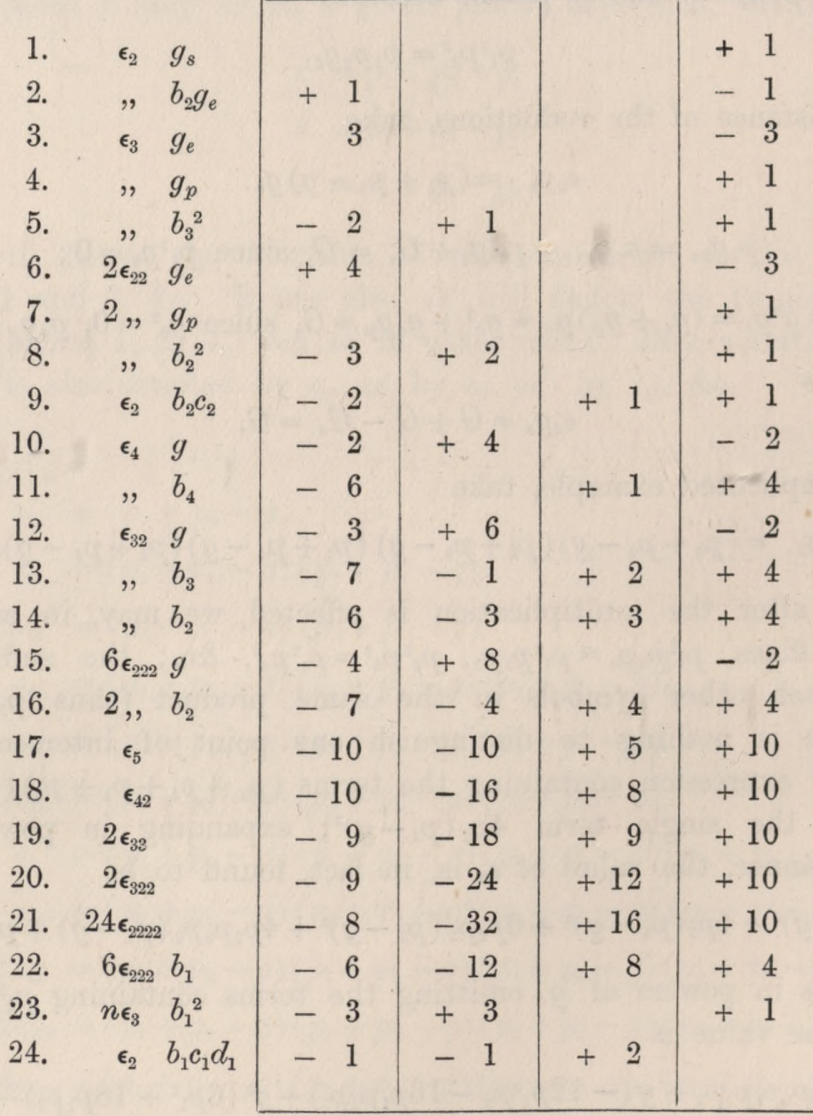


But in these formulæ $p_{1}^{2} p_{2}^{2}, p_{1}^{2} p_{2} p_{3}, p_{1} p_{2} p_{3} p_{4}, G$ have numerical values which are different according to the number of points of intersection presenting themselves in the several formulæ; viz. this number being called $i$, we have for the formulæ in

$$
\begin{array}{rccccccccccccc}
\epsilon_{2} & \epsilon_{3} & \epsilon_{22} & \epsilon_{4} & \epsilon_{32} & \epsilon_{222} & \epsilon_{5} & \epsilon_{42} & \epsilon_{33} & \epsilon_{322} & \epsilon_{2222} & \epsilon_{222} b_{1} & \epsilon_{3} b_{1}{ }^{2} & \epsilon_{2} b_{1} c_{1} d_{1} \\
i=2 & 3 & 4 & 4 & 5 & 6 & 5 & 6 & 6 & 7 & 8 & 7 & 4 & 5,
\end{array}
$$

and the values of the symbols are

$$
\begin{array}{llr}
p_{1}^{2} p_{2}{ }^{2} & =n^{2}(n-2)(n-3) & \ldots(n-i+1), \\
p_{1}{ }^{2} p_{2} p_{3} & =n^{2}(n-1)(n-3) & \ldots(n-i+1), \\
p_{1} p_{2} p_{3} p_{4} & =n^{2}\left(2 n^{2}-6 n+3\right)(n-4) \ldots(n-i+1), \\
G & =n(n-1)(n-2) & \ldots(n-i+1) .
\end{array}
$$

Thus, suppose $i=4$, the subject is a line bearing the points $1,2,3,4$, which are intersections of the line with the surface $F$; we have then $G$ as the condition in order that this line (or, say, the line of the subject) may coincide with a given line, which given line intersects the surface in $n$ points; any four of these (their order being attended to) may be regarded as being the points $1,2,3,4$; or there are $n(n-1)(n-2)(n-3)$ subjects satisfying the prescribed condition (that the line of the subject may coincide with the given line). Hence here $G=n(n-1)(n-2)(n-3)$; and so in general $G=n(n-1)(n-2) \ldots(n-i+1)$.

Next, for $p_{1}^{2} p_{2}{ }^{2}$. Here $p_{1}{ }^{2}$ is the condition that the point 1 shall lie in each of two given planes, that is, in a given line, say $L_{1}$; and, similarly, $p_{2}{ }^{2}$ is the condition that 2 may lie in a given line $L_{2}$. We take any one of the $n$ intersections of $L_{1}$ with $F$ for the point 1 , and any one of the $n$ intersections of $L_{2}$ with $F$ for the point 2 ; this determines the line of the subject, but the $i-2$ points $3,4, \ldots, i$ are then any $i-2$ of the remaining $n-2$ intersections of this line with $F$; that is, $p_{1}^{2} p_{2}^{2}=n^{2}(n-2)(n-3) \ldots(n-i+1)$ as above.

Again, for $p_{1}^{2} p_{2} p_{3}$. Here $p_{1}{ }^{2}$ is the condition that 1 shall lie in a given line $L_{1}$; we therefore take for 1 any one of the $n$ intersections of $L_{1}$ with $F ; p_{2}$ is the condition that 2 may lie in a given plane $P_{2}$, it lies therefore in the curve of intersection of $P_{2}$ with $F$; and, similarly, 3 lies in the curve of intersection of a plane $P_{3}$ with $F$; the two planes intersect in a line meeting $F$ in $n$ points $\sigma$, and the two cones, vertex 1, which stand upon the plane curves respectively, intersect in the $n$ lines joining 1 with the $n$ points $\sigma$, and in $n^{2}-n$ other lines. The line of the subject is then any one of these $n^{2}-n$ lines, or, since the vertex is any one of $n$ points, the line is any one of $n\left(n^{2}-n\right),=n^{2}(n-1)$ lines; the remaining points $4,5, \ldots, i$ are any $i-3$ of the remaining $n-3$ intersections of the line with $F$; hence the formula

$$
p_{1}^{2} p_{2} p_{3}=n^{2}(n-1)(n-3)(n-4) \ldots(n-i+1) .
$$

For $p_{1} p_{2} p_{3} p_{4}$. We have here $1,2,3,4$ lying in given plane sections of the surface $F$, and we have consequently to find the number of lines which can be drawn to meet each of these four sections. Observing that any two of the sections meet in the $n$ 
intersections with $F$ of the line of intersection of their planes, the order of the scroll generated by the lines which meet three of the sections is $2 n^{3}-3 n^{2}$; this scroll meets the fourth section in $n\left(2 n^{3}-3 n^{2}\right),=2 n^{4}-3 n^{3}$ points; or we have this number of lines meeting each of the four sections. But among these are included $3 n^{2}(n-1)$ lines which have to be rejected, viz. the sections 1 and 4 meet in $n$ points, each of which is the vertex of cones through the sections 1 and 2 respectively; these cones meet in $n$ lines, which are to be disregarded, and in $n^{2}-n$ other lines, and we have thus $n\left(n^{2}-n\right)$, $=n^{2}(n-1)$ lines; and similarly from the intersections of 2 and 4 , and from the intersections of 3 and $4, n^{2}(n-1)$ and $n^{2}(n-1)$ lines, in all $3 n^{2}(n-1)$ lines. Hence the number of lines meeting the four sections is

$$
2 n^{4}-3 n^{3}-3 n^{3}+3 n^{2},=2 n^{4}-6 n^{3}+3 n^{2} ;
$$

taking any one of these for the line of the subject, the remaining points $5,6, \ldots, i$ are any $i-4$ of the remaining $n-4$ intersections, or we have the required formula

$$
p_{1} p_{2} p_{3} p_{4}=n^{2}\left(2 n^{2}-6 n+3\right)(n-4) \ldots(n-i+1) .
$$

The four numbers $p_{1}^{2} p_{2}^{2}, p_{1}^{2} p_{2} p_{3}, p_{1} p_{2} p_{3} p_{4}, G$ for any line of the table being now known, we can at once calculate the required values $\epsilon_{2} g_{s}$, \&c., as the case may be; for instance,

$$
\begin{aligned}
i=5, \quad \epsilon_{5}= & -10 p_{1}^{2} p_{2}{ }^{2} \quad=-10 n^{2}(n-2)(n-3)(n-4) \\
& -10 p_{1}{ }^{2} p_{2} p_{3} \quad-10 n^{2}(n-1)(n-3)(n-4) \\
& +5 p_{1} p_{2} p_{3} p_{4}+5 n^{2}\left(2 n^{2}-6 n+3\right)(n-4) \\
& +10 G \quad+10 n(n-1)(n-2)(n-3)(n-4) \\
& =5 n(n-4)\left({ }^{7} n-12\right) .
\end{aligned}
$$

In fact, throwing out $n(n-4)$, the remaining terms give

$$
\begin{aligned}
& -10 n^{3}+50 n^{2}-60 n \\
& -10 n^{3}+40 n^{2}-30 n \\
& +10 n^{3}-30 n^{2}+15 n \\
& +10 n^{3}-60 n^{2}+110 n-60 \\
& 35 n-60,=5(7 n-12) .
\end{aligned}
$$

And we obtain in like manner the other formulæ of the table.

The remainder of $\S 33$ contains investigations of less systematically connected theorems, and I quote the results only.

25. If on the surface $F_{n}$ there is a curve order $r$, then of the tangent planes of $F_{n}$ along this curve there pass $r(n-1)$ through an arbitrary point of space; aliter, class of torse is $=r(n-1)$.

In particular, for curve of 4-pointic contact, $r=n(11 n-24)$, class of torse is

$$
=n(n-1)(11 n-24) \text {. }
$$

No. of tangent planes through line, or class of surface, $=n(n-1)^{2}$. 
26. $\epsilon_{3} b_{3} g=\epsilon_{3} b_{3}{ }^{2}+\epsilon_{3} g_{e}=2 n+3 n(n-2),=n(3 n-4)$.

$\epsilon_{3} b_{3} g,=n(3 n-4)$, is the order of curve of contact of the 3-pointic (chief) tangents which meet a given line.

Parabolic tangents are coincident chief tangents.

No. of 4-pointic parabolic tangents $=2 n(n-2)(11 n-24)$.

27. Order of parabolic curve $=4 n(n-2)$.

Order of regulus formed by parabolic tangents

$$
=2 n(n-2)(3 n-4) \text {. }
$$

The parabolic curve and curve of contacts of an $\epsilon_{4}$ tangent meet in

$$
4 n(n-2)(11 n-24)
$$

points, i.e., they touch in $2 n(n-2)(11 n-24)$ points.

28. Umbilici. No. is $=2 n\left(5 n^{2}-14 n+11\right)$.

29. No. of points at which the chief tangents being distinct are each of them 4-pointic, or, what is the same thing, No. of actual double points of curve $\epsilon_{4}$,

$$
=5 n\left(7 n^{2}-28 n+30\right),
$$

$n=3$, No. is $15(63-84+30),=135$, viz. this is the number of points of intersection of two of the 27 lines; or, what is the same thing, the number of triple tangent planes is $=45$.

30. No. of parabolic tangents which have besides a 2-pointic contact is

$$
=2 n(n-2)(n-4)\left(3 n^{2}+5 n-24\right) \text {. }
$$

31. No. of double tangent planes such that line through points of contact is at one of these points 3-pointic

$$
=n(n-2)(n-4)\left(n^{3}+3 n^{2}+13 n-48\right) .
$$

32. No. of points where one chief tangent is 4-pointic, the other 3-pointic and (at another point of the surface) 2-pointic is

$$
=n(n-4)\left(27 n^{3}-13 n^{2}-264 n+396\right) .
$$

33. No. of points where chief tangents being distinct are each of them at another point of the surface 2-pointic is

$$
=n(n-4)\left(4 n^{5}-4 n^{4}-95 n^{3}+99 n^{2}+544 n-840\right) .
$$

34. The curve of contacts $b_{3}$ of an $\epsilon_{32}$ tangent has with the parabolic curve 2pointic intersections only, and these are at the points for which the chief tangent is (at another point of the surface) 2-pointic.

35. The curve of contacts $b_{3}$ of an $\epsilon_{32}$ tangent has, with the curve of contacts of an $\epsilon_{4}$ tangent, 2-pointic intersections at the contacts of an $\epsilon_{5}$ tangent; and has also simple intersections with the same curve, $1^{\circ}$ at the contacts $b_{4}$ of an $\epsilon_{42}$ tangent, $2^{\circ}$ at the points where the chief tangents are $\epsilon_{4}$ and $\epsilon_{32}$. 